\title{
In vitro comparison of nasal continuous positive airway pressure devices for neonates
}

\author{
A G De Paoli, C J Morley, P G Davis, R Lau, E Hingeley
}

Arch Dis Child Fetal Neonatal Ed 2002;86:F42-F45

See end of article for authors' affiliations

......................

Correspondence to: Dr De Paoli, Department of Neonatology, Royal

Women's Hospital, 132

Grattan Street, Carlton,

Victoria 3053, Australia; depaolit@

cryptic.rch.unimelb.edu.au

Accepted

8 January 2002

\begin{abstract}
Objective: To compare the resistance in vitro of different devices used for the delivery of nasal continuous positive airway pressure (NCPAP) in neonates.

Design: Flows of 4-8 litres/min were passed through a selection of neonatal NCPAP devices (single prong, Duotube, Argyle prong, Hudson prong, Infant Flow Driver), and the resultant fall in pressure measured using a calibrated pressure transducer.

Results: The decrease in pressure $\left(\mathrm{cm} \mathrm{H}_{2} \mathrm{O}\right)$ for each device (size in parentheses) at a constant flow of 6 litres/min was: Duotube: $(2.5), 21 ;(3.0), 6.2 ;(3.5), 2.3$; single prong: $(2.5), 4.4 ;(3.0), 2.1 ;(3.5)$, 1.2; Argyle prong: (XS), 3.6; (S), 1.9; (L), 1.5; Hudson prong: (0), 3.1; (1), 1.8; (2), 0.6; (3), 0.4; (4), 0.3 ; Infant Flow Driver: (small), 0.3; (medium), -0.3; (large), -0.5 .

Conclusions: A large variation in the potential fall in pressure may occur in the clinical setting. Devices with short double prongs had the lowest resistance to flow. These results have implications in the selection of the optimal device/s for clinical application and for future comparisons in randomised trials of NCPAP in neonates.
\end{abstract}

C ontinuous positive airway pressure (CPAP) was first used to support the breathing of neonates in the early 1970s. The initial mode of delivery through an endotracheal tube or headchamber ${ }^{1}$ was superseded by the use of nasal prongs which could deliver CPAP in a simpler and less invasive manner. ${ }^{23}$

Nasal continuous positive airway pressure (NCPAP) is now an established mode of respiratory support for neonates in the period after extubation ${ }^{4}$ and is often used as the primary treatment for respiratory distress ${ }^{5}$ and apnoea of prematurity. ${ }^{67}$ As a consequence of further developments ${ }^{8-11}$ and a continuing clinical need, there is now a broad range of prongs used to deliver NCPAP. There is little information to guide clinicians or researchers seeking to compare the effectiveness of these devices.

One important characteristic of a NCPAP device is its resistance to airflow. This determines the fall in pressure from that measured in the delivery circuit to that transmitted to the respiratory tract. Although previous work has analysed the resistance of neonatal endotracheal tubes, ${ }^{12}{ }^{13}$ there have been no direct comparisons of the resistance of NCPAP devices. Our objective was to measure the pressure drop across a variety of NCPAP devices used in current neonatal practice.

\section{METHODS}

We selected a series of NCPAP devices of different sizes currently used in neonatal intensive care units. These were:

- Hudson prong (Hudson-RCI, Temecula, California, USA), sizes 0 to 4 ;

- Argyle prong (Sherwood Medical, St Louis, Missouri, USA), sizes large (L), small (S), and extra small (XS);

- Flow Driver generator (EME, Brighton, UK), prong sizes small, medium, and large;

- Duotube (Parker Medical, Melbourne, Australia), sizes 2.5, 3.0, and $3.5 \mathrm{~mm}$ (outer diameter);

- Mallinckrodt endotracheal tube (Mallinckrodt Medical, Athlone, Ireland) cut to a length of $5 \mathrm{~cm}$ (internal diameters of 2.5, 3.0, and $3.5 \mathrm{~mm}$ ) with a connector (RPS; Sims, California, USA).
Prong dimensions (table 1) were measured using digital calipers accurate to $\pm 0.03 \mathrm{~mm}$.

Flows of 4-8 litres/min were applied to the device so that the entire flow passed through the prong/s. These flows were selected because they encompass the typical range of flows observed to flow through Hudson prongs in our unit. The flow was measured using a Platon flow meter (G A Platon Ltd, Sheffield, UK) with a range of $0-10$ litres/min and an accuracy of $\pm 1.25 \%$ of full scale. Air was used for all measurements, and room temperature during the study varied from 24 to $26^{\circ} \mathrm{C}$. Figure 1 shows the apparatus connected to a single prong.

The pressure generated by the resistance to flow in each device (pressure drop) was measured using a Sensym pressure transducer (Sensortechnics, Puchheim, Germany) with an operating range of $0-13 \mathrm{~cm} \mathrm{H}_{2} \mathrm{O}$ and a typical combined accuracy of $\pm 0.2 \%$ of full scale. The transducer was connected to the pressure port of the device, if one was present, or to a pressure port on a connector (with negligible intrinsic resistance at these flows) attached directly to the device. The pressure was measured twice at each flow, with variation between values in the transducer operating range not exceeding $0.1 \mathrm{~cm}$ $\mathrm{H}_{2} \mathrm{O}$ and typically less than $0.05 \mathrm{~cm} \mathrm{H}_{2} \mathrm{O}$. The transducer was calibrated before each study using a water manometer. The analogue output from the pressure transducer was amplified (PC Polygraph HR; Synectics Medical, Stockholm, Sweden), digitised, and recorded using Spectra software. ${ }^{14}$ Pressures greater than $15 \mathrm{~cm} \mathrm{H}_{2} \mathrm{O}$ have been rounded to the nearest $\mathrm{cm}$ $\mathrm{H}_{2} \mathrm{O}$.

The bar graph (fig 2) was created using SPSS. ${ }^{15}$ The study was carried out in the Department of Neonatology at the Royal Women's Hospital, Melbourne, Australia.

\section{RESULTS}

Table 1 summarises the device dimensions and decrease in pressure measured on passing different flows through the

Abbreviations: CPAP, continuous positive airway pressure; NCPAP nasal continuous positive airway pressure. 
Table 1 Dimensions of nasal continuous positive airway pressure devices and pressure drop with various flows

\begin{tabular}{|c|c|c|c|c|c|c|c|c|c|}
\hline \multirow[b]{2}{*}{ Device } & \multirow[b]{2}{*}{ Size } & \multirow{2}{*}{$\begin{array}{l}\text { Prong } \\
\text { length† }(\mathrm{mm})\end{array}$} & \multirow{2}{*}{$\begin{array}{l}\text { Internal } \\
\text { diameter } \neq(\mathrm{mm})\end{array}$} & \multirow{2}{*}{$\begin{array}{l}\text { Outer } \\
\text { diameter } \neq(\mathrm{mm})\end{array}$} & \multicolumn{5}{|c|}{ Pressure drop $\left(\mathrm{cm} \mathrm{H}_{2} \mathrm{O}\right)$ at various flows } \\
\hline & & & & & $4 \mathrm{I} / \mathrm{min}$ & $5 \mathrm{l} / \mathrm{min}$ & $6 \mathrm{l} / \mathrm{min}$ & $7 \mathrm{l} / \mathrm{min}$ & $81 / \mathrm{min}$ \\
\hline \multirow[t]{3}{*}{ Duotube } & 2.5 & 40 & 1.4 & 2.5 & 9.7 & 15 & 21 & 29 & 38 \\
\hline & 3.0 & 40 & 1.8 & 3.0 & 3.0 & 4.5 & 6.2 & 8.3 & 10.5 \\
\hline & 3.5 & 40 & 2.5 & 3.5 & 1.1 & 1.7 & 2.3 & 3.1 & 3.9 \\
\hline \multirow{3}{*}{ Single prong* } & 2.5 & 50 & 2.5 & 3.8 & 2.2 & 3.3 & 4.4 & 5.9 & 7.3 \\
\hline & 3.0 & 50 & 3.0 & 4.3 & 1.1 & 1.5 & 2.1 & 2.7 & 3.3 \\
\hline & 3.5 & 50 & 3.5 & 4.9 & 0.6 & 0.9 & 1.2 & 1.6 & 1.9 \\
\hline \multirow[t]{3}{*}{ Argyle prong } & Extra small & 6 & 1.8 & 3.1 & 1.7 & 2.6 & 3.6 & 4.8 & 6.2 \\
\hline & Small & 8 & 2.3 & 4.0 & 0.9 & 1.4 & 1.9 & 2.5 & 3.2 \\
\hline & Large & 10 & 2.3 & 4.8 & 0.7 & 1.1 & 1.5 & 2.1 & 2.6 \\
\hline \multirow[t]{5}{*}{ Hudson prong } & 0 & 11 & 2.3 & 3.7 & 1.4 & 2.2 & 3.1 & 4.2 & 5.4 \\
\hline & 1 & 11 & 2.6 & 3.9 & 0.8 & 1.3 & 1.8 & 2.5 & 3.3 \\
\hline & 2 & 11 & 3.0 & 4.6 & 0.3 & 0.5 & 0.6 & 0.9 & 1.1 \\
\hline & 3 & 12 & 3.5 & 4.9 & 0.2 & 0.3 & 0.4 & 0.5 & 0.6 \\
\hline & 4 & 15 & 4.0 & 5.4 & 0.1 & 0.2 & 0.3 & 0.5 & 0.6 \\
\hline \multirow[t]{3}{*}{ Infant Flow Driver } & Small & 6 & 3.0 & 3.9 & 0.2 & 0.2 & 0.3 & 0.4 & 0.5 \\
\hline & Medium & 7 & 3.5 & 4.3 & -0.1 & -0.2 & -0.3 & -0.4 & -0.5 \\
\hline & Large & 7 & 4.0 & 4.6 & -0.2 & -0.3 & -0.5 & -0.7 & -0.9 \\
\hline
\end{tabular}

* Refers to Mallinckrodt endotracheal tube cut down to $5 \mathrm{~cm}$ length attached to standard endotracheal tube adapter with pressure side port. $†$ Does not include the length proximal to the bridge between the prongs. Ilnternal and outer diameters were measured at the prong tip.

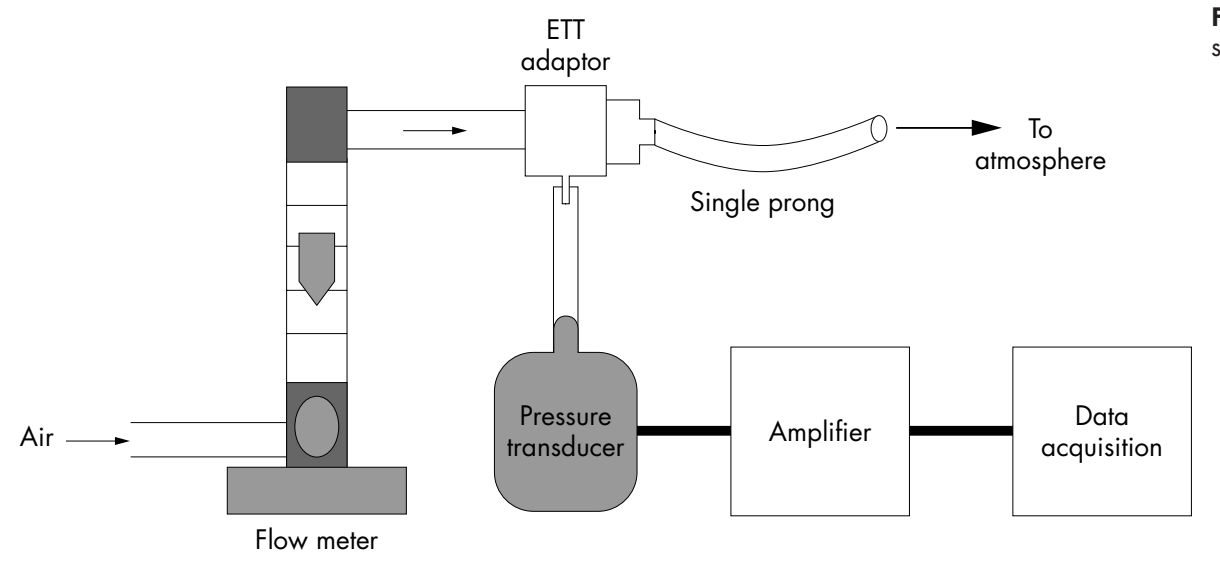

Figure 1 Apparatus connected to a single prong device.

NCPAP devices. Figure 2 shows the pressure measured in the devices at a constant flow of 6 litres/min represented as a bar graph.

Of all the double prong devices, the Infant Flow Driver, medium and large sizes, showed the lowest decrease in pressure, with increasing flows generating an increasingly slightly negative pressure. The pressure generated in the Infant Flow Driver with small prongs was equivalent to that generated in the size 4 Hudson prong. The largest falls in pressure were evident in the size 2.5 and 3.0 Duotube.

The single prong device (Mallinkrodt tube plus connector), at a length of $5 \mathrm{~cm}$ and internal diameter of $2.5 \mathrm{~mm}$, showed a greater fall in pressure than all the short double prongs assessed. The small Argyle prong was equivalent to the size 1 Hudson prong, and the extra small Argyle prong showed a large pressure drop, smaller only than the Duotubes and the single prong of internal diameter $2.5 \mathrm{~mm}$.

\section{DISCUSSION}

Resistance to flow is a significant factor when selecting the most appropriate device to deliver NCPAP. However, data on the impact of different NCPAP devices on clinical outcomes and physiological parameters are limited.

A study by Goldman et al $^{16}$ showed the increased resistance and imposed work of breathing with Argyle prongs compared with a face mask for the delivery of CPAP to neonates. Courtney et $a l^{17}$ examined the effect of three different nasal devices on lung volume, showing better recruitment when using increased flow, via the Flow Driver system, to increase pressure compared with fixed flows through Inca prongs and modified nasal cannulae. A short term randomised study comparing the Flow Driver system with conventional nasal prongs showed no difference in the incidence of apnoea, bradycardia, and desaturations. ${ }^{18}$ Others compared the Flow Driver system with the single nasal prong and found no significant difference in various physiological parameters over short time periods. ${ }^{19} 20$

Randomised trials have compared the impact of different double nasal prong devices on rates of extubation failure in preterm neonates. ${ }^{21}{ }^{22}$ Other randomised trials have shown a reduced rate of extubation failure with use of double nasal prong devices compared with single nasal prongs. ${ }^{23}{ }^{24}$

Although the resistance of a NCPAP device is important, other factors may contribute to the effectiveness of different devices in the neonatal intensive care setting. These include the characteristics of the pressure source. Underwater bubble CPAP, for example, delivers to the circuit an oscillatory pressure waveform that may have its own clinical effect. The ease of use, patient comfort, and avoidance of trauma to the 


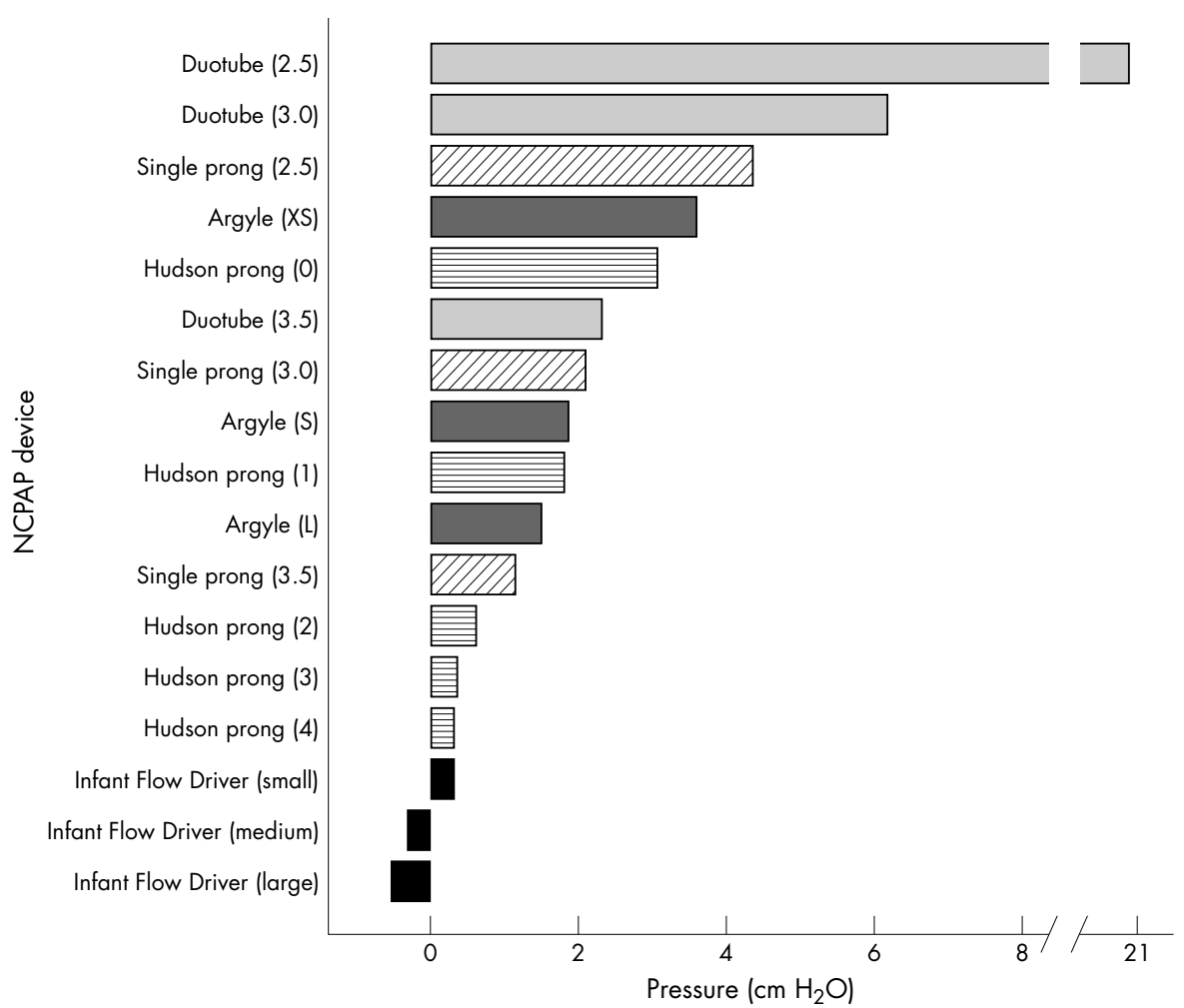

Figure 2 Pressure measured in nasal continuous positive airway pressure (NCPAP) devices at a flow rate of 6 litres/min. Device sizes are given in parentheses.

nares and columella ${ }^{25} 26$ when nasal prongs are used are important factors which also require further evaluation.

Although other factors must be considered, we have shown that there is significant variation in resistance between different nasal prongs, which is likely to result in different degrees of transmission to the airway if the pressures and flows in the CPAP delivery system are equal. Resistance in the medium and large sized Infant Flow Driver prongs is sufficiently low to create a negative pressure within the device by virtue of the Venturi effect. The fall in pressure in the size 2.5 and 3.0 Duotube is high relative to the other devices, even at low flows. Despite these findings, it is important to note that they do not establish the clinical superiority of one device over another.

Further studies using meaningful clinical outcomes are required to define the optimal method and interface for NCPAP administration. We recommend comparisons in future trials of the short, double prong devices with low resistance identified in this study.

\section{ACKNOWLEDGEMENTS}

This study was supported by: the Royal Women's Hospital Foundation and the Division of Research and Education, Royal Women's Hospital, Melbourne; Murdoch Children's Research Institute.

\section{Authors' affiliations}

A G De Paoli, C J Morley, P G Davis, R Lau, Neonatal Services, Royal Women's Hospital, Carlton, Victoria, Australia

E Hingeley, Biomedical Engineering Department, Royal Women's Hospital

\section{REFERENCES}

1 Gregory GA, Kitterman JA, Phibbs RH, et al. Treatment of the idiopathic respiratory-distress syndrome with continuous positive airway pressure. $N$ Engl J Med 1971;284:1333-40.

2 Kattwinkel J, Fleming D, Cha CC, et al. A device for administration of continuous positive airway pressure by the nasal route. Pediatrics 1973;52:131.
3 Novogroder M, MacKuanying N, Eidelman Al, et al. Nasopharyngeal ventilation in respiratory distress syndrome. J Pediatr 1973;82:1059-62.

4 Davis PG, Henderson-Smart DJ. Nasal continuous positive airways pressure immediately after extubation for preventing morbidity in preterm infants. In: The Cochrane Library. Oxford: Update Software, 2001 ; Issue

5 Subramaniam P, Henderson-Smart DJ, Davis PG. Prophylactic nasal continuous positive airways pressure for preventing morbidity and mortality in very preterm infants. In: The Cochrane Library. Oxford: Update Software, 2001; Issue 1.

6 Kattwinkel J, Nearman HS, Fanaroff AA, et al. Apnea of prematurity, comparative therapeutic effects of cutaneous stimulation and nasal continuous positive airway pressure. J Pediatr 1975;86:588-92.

7 Speidel BD, Dunn PM. Use of nasal continuous positive airway pressure to treat severe recurrent apnoea in very preterm infants. Lancet 1976;2:658-60.

8 Caliumi-Pellegrini G, Agostino R, Orzalesi $M$, et al. Twin nasal cannula for administration of continuous positive airway pressure to newborn infants. Arch Dis Child 1974;49:228-30

9 Wung J, Driscoll Jr JM, Epstein RA, et al. A new device for CPAP by nasal route. Crit Care Med 1975;3:76-8.

10 Field $\mathbf{D}$, Vyas $H$, Milner $A D$, et al. Continuous positive airway pressure via a single nasal catheter in preterm infants. Early Hum Dev 1985:11:275-80.

11 Moa G, Nilsson K, Zetterström H, et al. A new device for administration of nasal continuous positive airway pressure in the newborn: An experimental study. Crit Care Med 1988;16:1238-42.

12 Wall MA. Infant endotracheal tube resistance: effects of changing length diameter, and gas density. Crit Care Med 1980;8:38-40.

13 Jarreau P, Louis B, Dassieu G, et al. Estimation of inspiratory pressure drop in neonatal and pediatric endotracheal tubes. J Appl Physiol 1999;87:36-46.

14 Spectra Physiological Software [computer program]. London: Grove Medical Ltd, 2001; version 3.004.

15 SPSS for Windows [computer program]. SPSS I. 10.0.7. 2000.

16 Goldman SL, Brady JP, Dumpit FM. Increased work of breathing associated with nasal prongs. Pediatrics 1979;64:160-4

17 Courtney SE, Pyon KH, Saslow JG, et al. Lung recruitment and breathing pattern during variable versus continuous flow nasal continuous positive airway pressure in premature infants: an evaluation of three devices. Pediatrics 2001;107:304-8.

18 Telenko T, Peliowski A, Hudson-Mason A. Continuous positive airway pressure (CPAP) in the treatment of apnoea of prematurity: a comparison of two CPAP delivery systems [Abstract]. Pediatr Res 1999;45:228A.

19 Kavvadia V, Greenough A, Dimitriou G. Effect on lung function of continuous positive airway pressure administered either by infant flow driver or a single nasal prong. Eur J Pediatr 2000;159:289-92.

20 Ahluwalia JS, White DK, Morley CJ. Infant flow driver or single prong nasal continuous positive airway pressure: short-term physiological effects. Acta Paediatr 1998;87:325-7. 
21 Murphy WP, Hansell BJ, Fuloria $M$, et al. A randomized comparison of two CPAP systems for the successful extubation of extremely low birth weight infants [Abstract]. Pediatr Res 2001;49:288A.

22 Sun SC, Tien HC. Randomized controlled trial of two methods of nasal CPAP (NCPAP): flow driver vs conventional NCPAP [Abstract]. Pediatr Res 1999:45:322A.

23 Davis PG, Davies MW, Faber BM. A randomized controlled trial of two methods of delivering nasal continuous positive airway pressure after extubation to infants less than 1000g: binasal (Hudson) versus single nasal prongs Arch Dis Child Fetal Neonatal Ed 2001;85:F82-5.
24 Roukema H, O'Brien K, Nesbitt $\mathrm{K}$, et al. A randomized controlled trial of infant flow continuous positive airway pressure (CPAP) versus nasopharyngeal CPAP in the extubation of babies $<=1250 \mathrm{~g}$ [Abstract]. Pediatr Res 1999;45:318A.

25 Loftus BC, Ahn J, Haddad Jr J. Neonatal nasal deformities secondary to nasal continuous positive airway pressure. Laryngoscope 1994; 104:1019-22.

26 Robertson NJ, McCarthy LS, Hamilton PA, et al. Nasal deformities resulting from flow driver continuous positive airway pressure. Arch Dis Child Fetal Neonatal Ed 1996;75:F209-12.

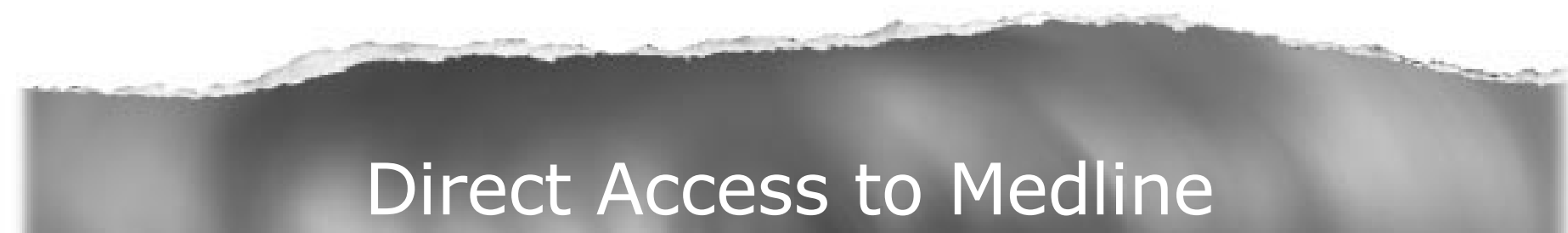

\section{Medline}

Link to Medline from the homepage and get straight into the National Library of Medicine's premier bibliographic database. Medline allows you to search across 9 million records of bibliographic citations and author abstracts from approximately 3,900 current biomedical journals.

www.archdischild.com 